\title{
ON THE EXISTENCE OF MAXIMIZERS FOR A FAMILY OF RESTRICTION THEOREMS
}

\author{
LUCA FANELLI, LUIS VEGA, AND NICOLA VISCIGLIA
}

\begin{abstract}
We prove the existence of maximizers for a general family of restrictions operators, up to the end-point. We also provide some counterxamples in the end-point case.
\end{abstract}

In the sequel we shall denote by $d \mu$ any positive measure on $\mathbb{R}_{\xi}^{d}$. For every fixed $d \mu$ we define

$$
T_{\mu}: C^{0}(\operatorname{supp}(d \mu)) \ni \hat{h}(\xi) \rightarrow \int e^{i x \cdot \xi} \hat{h}(\xi) d \mu \in \mathcal{C}^{\infty}\left(\mathbb{R}_{x}^{d}\right)
$$

Given two Banach spaces $X, Y$ we denote by $\mathcal{L}(X, Y)$ the space of linear and continuous operators between $X$ and $Y$.

Definition 0.1. A measure $d \mu$ on $\mathbb{R}_{\xi}^{d}$ satisfies the restriction condition w.r.t. $p \in$ $[1, \infty]$ (shortly $\left.(R C)_{p}\right)$ provided that $T_{\mu} \in \mathcal{L}\left(L^{2}(d \mu), L^{p}\left(\mathbb{R}_{x}^{d}\right)\right)$.

Definition 0.2. Assume that $d \mu$ satisfies $(R C)_{p}$ then we say that there is a maximizer for $T_{\mu}$ w.r.t. $p$ provided that there exists $\hat{h} \in L^{2}(d \mu)$ such that:

$$
\|\hat{h}\|_{L^{2}(d \mu)}=1
$$

and

$$
\left\|T_{\mu} \hat{h}\right\|_{L^{p}\left(\mathbb{R}_{x}^{d}\right)}=\left\|T_{\mu}\right\|_{\mathcal{L}\left(L^{2}(d \mu), L^{p}\left(\mathbb{R}_{x}^{d}\right)\right)} .
$$

Definition 0.3. Assume that $d \mu$ satisfies $(R C)_{p}$ then we say that $\hat{h}_{n} \in L^{2}(d \mu)$ is a maximizing sequence for $T_{\mu}$ w.r.t. $p$ provided that:

$$
\left\|\hat{h}_{n}\right\|_{L^{2}(d \mu)}=1
$$

and

$$
\lim _{n \rightarrow \infty}\left\|T_{\mu} \hat{h}_{n}\right\|_{L^{p}\left(\mathbb{R}_{x}^{d}\right)}=\left\|T_{\mu}\right\|_{\mathcal{L}\left(L^{2}(d \mu), L^{p}\left(\mathbb{R}_{x}^{d}\right)\right)}
$$

We have the following

Theorem 0.1. Let $d \mu$ be a positive compactly supported measure on $\mathbb{R}_{\xi}^{d}$ and let

$$
p_{0}(\mu)=\inf \left\{1 \leq p \leq \infty \mid(R C)_{p} \text { holds for } d \mu\right\} .
$$

Then for every

$$
\max \left\{2, p_{0}(\mu)\right\}<p \leq \infty
$$

there exists a maximizer for $T_{\mu}$ w.r.t. $p$. More precisely for every maximizing sequence $\hat{h}_{n}(\xi)$ for $T_{\mu}$ w.r.t. $p$, there exists $x_{n} \in \mathbb{R}^{d}$ such that $e^{i x_{n} \cdot \xi} \hat{h}_{n}(\xi)$ is compact in $L^{2}(d \mu)$.

2000 Mathematics Subject Classification. 35L05, 58J45.

Key words and phrases. Fourier restriction Theorems, Strichartz estimates. 
In order to treat the case $p \neq \infty$ we shall use the following general fact whose proof is inspired by $([1],[4])$.

Proposition 0.1. Let $\mathcal{H}$ be a Hilbert space and $T \in \mathcal{L}\left(\mathcal{H}, L^{p}\left(\mathbb{R}^{d}\right)\right)$ for a suitable $p \in(2, \infty)$. Let $\left\{h_{n}\right\}_{n \in \mathbb{N}} \in \mathcal{H}$ such that:

(1) $\left\|h_{n}\right\|_{\mathcal{H}}=1$;

(2) $\lim _{n \rightarrow \infty}\left\|T h_{n}\right\|_{L^{p}\left(\mathbb{R}^{d}\right)}=\|T\|_{\mathcal{L}\left(\mathcal{H}, L^{p}\left(\mathbb{R}^{d}\right)\right)} ;$

(3) $h_{n} \rightarrow \bar{h} \neq 0$

(4) $T\left(h_{n}\right) \rightarrow T(\bar{h})$ a.e. in $\mathbb{R}^{d}$.

Then $h_{n} \rightarrow \bar{h}$ in $\mathcal{H}$, in particular $\|\bar{h}\|_{\mathcal{H}}=1$ and $\|T(\bar{h})\|_{L^{p}\left(\mathbb{R}^{d}\right)}=\|T\|_{\mathcal{L}\left(\mathcal{H}, L^{p}\left(\mathbb{R}^{d}\right)\right)}$.

Remark 0.1. The main difference between Proposition 0.1 and Lemma 2.7 in [4] is that we only need to assume weak convergence in the Hilbert space $\mathcal{H}$ for the maximizing sequence $h_{n}$. On the other hand the argument in [4] works for operators defined between general Lebesgue spaces and not necessarily in the Hilbert spaces framework.

Remark 0.2 . We shall use Proposition 0.1 by choosing $\mathcal{H}=L^{2}(d \mu)$. The main point is that in the assumptions of Proposition 0.1 we do not assume a-priori the almost everywhere convergence of the maximizing sequence (which in our concrete context cannot be easily checked).

Next result shows that in general Theorem 0.1 cannot be extended to the endpoint case $p=p_{0}(\mu)$.

For every $M>0$ we consider the compactly supported measures:

$$
\begin{gathered}
d \mu_{M}^{1}=\delta_{P_{M}^{1}}, P_{M}^{1}=\left\{\left(\xi,|\xi|^{2}\right), \xi \in \mathbb{R},|\xi| \leq M\right\} ; \\
d \mu_{M}^{2}=\delta_{P_{M}^{2}}, P_{M}^{2}=\left\{\left(\xi,|\xi|^{2}\right), \xi \in \mathbb{R}^{2},|\xi| \leq M\right\} ; \\
d \sigma_{M}=\frac{1}{\sqrt{|\xi|}} \delta_{C_{M}}, C_{M}=\cup_{ \pm}\left\{(\xi, \pm|\xi|), \xi \in \mathbb{R}^{3},|\xi| \leq M\right\}
\end{gathered}
$$

where we have denoted in general by $\delta_{S}$ the flat measure on $S$.

Remark 0.3. Notice that the restriction operators associated to the measures $d \mu_{M}^{1}$, $d \mu_{M}^{2}, d \sigma_{M}$ are strictly related to the Strichartz estimates associated respectively to the Schrödinger equation in 1-D, 2-D and to the wave equation in 3-D (provided that the initial data are localized in frequencies).

We have the following

Theorem 0.2. The condition $(R C)_{6}$ holds for $d \mu_{M}^{1}$ and $(R C)_{4}$ holds for $d \mu_{M}^{2}$ and $d \sigma_{M}$ for every $0<M \leq \infty$. However there are not maximizers for

$$
T_{\mu_{M}^{1}}, T_{\mu_{M}^{2}}, T_{\sigma_{M}}
$$

w.r.t. to $p=6, p=4, p=4$ (respectively) provided that $M \neq \infty$.

Remark 0.4. In [2] it is proved the existence of maximizers for the restriction on the sphere $\mathcal{S}^{2}$ w.r.t. to $p=4$ (which turns out to be the end-point value for the restriction on $\mathcal{S}^{2}$ ). In the best of our knowledge this is the unique result concerning existence of maximizers for the end-point restriction problem on a compact manifold. 


\section{Proof of Theorem 0.2}

We work with $d \mu_{M}^{1}$ (the same argument works for $d \mu_{M}^{2}$ and $d \sigma_{M}$ ). Notice that validity of $(R C)_{6}$ for $d \mu_{M}^{1}$ follows from the usual Strichartz estimates

$$
\left\|e^{i t \Delta} f\right\|_{L^{6}\left(\mathbb{R}^{2}\right)} \leq C\|f\|_{L^{2}(\mathbb{R})}
$$

Moreover the maximization problem

$$
\sup _{\|\hat{g}\|_{L^{2}\left(d \mu_{M}^{1}\right)=1}}\left\|T_{\mu_{M}^{1}}(\hat{g})\right\|_{L^{6}\left(\mathbb{R}^{2}\right)}
$$

is equivalent to

$$
\sup _{\|h\|_{L^{2}(\mathbb{R})=1, \text { supph } \hat{h}(\xi) \subset(-M, M)}}\left\|e^{i t \Delta} h\right\|_{L^{6}\left(\mathbb{R}^{2}\right)} .
$$

On the other hand by an elementary rescaling argument we get:

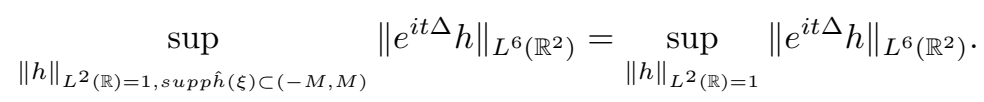

By the previous identity it is easy to deduce that if a maximizer exists for (1.1) then it is necessarily a maximizer for

$$
\sup _{\|h\|_{L^{2}(\mathbb{R})=1}}\left\|e^{i t \Delta} h\right\|_{L^{6}\left(\mathbb{R}^{2}\right)}
$$

but this is absurd since by [3] there are no maximizers for (1.3) which are compactly supported in the Fourier variables.

\section{Proof of Proposition 0.1 and Theorem 0.1}

Proof of Prop 0.1 By using the Brézis and Lieb Lemma (see [1]) we get:

$$
\left\|T\left(h_{n}\right)-T(\bar{h})\right\|_{L^{p}\left(\mathbb{R}^{d}\right)}^{p}=\left\|T\left(h_{n}\right)\right\|_{L^{p}\left(\mathbb{R}^{d}\right)}^{p}-\|T(\bar{h})\|_{L^{p}\left(\mathbb{R}^{d}\right)}^{p}+o(1)
$$

and by the hypothesis (3) in the Proposition we get

$$
\left\|h_{n}-\bar{h}\right\|_{\mathcal{H}}^{2}=\left\|h_{n}\right\|_{\mathcal{H}}^{2}-\|\bar{h}\|_{\mathcal{H}}^{2}+o(1)
$$

In particular since $h_{n}$ is by hypothesis a maximizing sequence for $T$ we get

$$
\begin{gathered}
\|T\|_{\mathcal{L}\left(\mathcal{H}, L^{p}\left(\mathbb{R}^{d}\right)\right)}^{2}=\frac{\left(\left\|T\left(h_{n}\right)-T(\bar{h})\right\|_{L^{p}\left(\mathbb{R}^{d}\right)}^{p}+\|T(\bar{h})\|_{L^{p}\left(\mathbb{R}^{d}\right)}^{p}+o(1)\right)^{\frac{2}{p}}}{\left\|h_{n}-\bar{h}\right\|_{\mathcal{H}}^{2}+\|\bar{h}\|_{\mathcal{H}}^{2}+o(1)} \\
\leq \frac{\left(\left\|T\left(h_{n}\right)-T(\bar{h})\right\|_{L^{p}\left(\mathbb{R}^{d}\right)}^{2}+\|T(\bar{h})\|_{L^{p}\left(\mathbb{R}^{d}\right)}^{2}+o(1)\right)}{\left\|h_{n}-\bar{h}\right\|_{\mathcal{H}^{\prime}}^{2}+\|\bar{h}\|_{\mathcal{H}}^{2}+o(1)}
\end{gathered}
$$

where we have used the inequality

$$
(a+b+c)^{t} \leq a^{t}+b^{t}+c^{t} \forall a, b, c>0
$$

provided that $t \leq 1$. The estimate above implies

$$
\|T\|_{\mathcal{L}\left(\mathcal{H}, L^{p}\left(\mathbb{R}^{n}\right)\right)}^{2} \leq \frac{\left(\|T\|_{\mathcal{L}\left(\mathcal{H}, L^{p}\left(\mathbb{R}^{n}\right)\right)}^{2}\left\|h_{n}-\bar{h}\right\|_{L^{p}\left(\mathbb{R}^{n}\right)}^{2}+\|T(\bar{h})\|_{L^{p}\left(\mathbb{R}^{n}\right)}^{2}+o(1)\right)}{\left\|h_{n}-\bar{h}\right\|_{\mathcal{H}}^{2}+\|\bar{h}\|_{\mathcal{H}}^{2}+o(1)}
$$

and hence

$$
\begin{gathered}
\|T\|_{\mathcal{L}\left(\mathcal{H}, L^{p}\left(\mathbb{R}^{d}\right)\right)}^{2}\left(\left\|h_{n}-\bar{h}\right\|_{\mathcal{H}}^{2}+\|\bar{h}\|_{\mathcal{H}}^{2}+o(1)\right) \\
\leq\left(\|T\|_{\mathcal{L}\left(\mathcal{H}, L^{p}\left(\mathbb{R}^{d}\right)\right)}^{2}\left\|h_{n}-\bar{h}\right\|_{L^{p}\left(\mathbb{R}^{d}\right)}^{2}+\|T(\bar{h})\|_{L^{p}\left(\mathbb{R}^{d}\right)}^{2}+o(1)\right)
\end{gathered}
$$


which is equivalent to

$$
\begin{gathered}
\|T\|_{\mathcal{L}\left(\mathcal{H}, L^{p}\left(\mathbb{R}^{d}\right)\right)}^{2}\left(\|\bar{h}\|_{\mathcal{H}}^{2}+o(1)\right) \\
\leq\left(\|T(\bar{h})\|_{L^{p}\left(\mathbb{R}^{d}\right)}^{2}+o(1)\right) .
\end{gathered}
$$

In particular the previous estimate implies $\|T\|_{\mathcal{L}\left(\mathcal{H}, L^{p}\left(\mathbb{R}^{d}\right)\right)}^{2} \leq\left\|T\left(\frac{\bar{h}}{\|h\|_{\mathcal{H}}}\right)\right\|_{L^{p}\left(\mathbb{R}^{d}\right)}^{2}$ and due to the definition of $\|T\|_{\mathcal{L}\left(\mathcal{H}, L^{p}\left(\mathbb{R}^{d}\right)\right)}$ it implies easily the following

$$
\|T\|_{\mathcal{L}\left(\mathcal{H}, L^{p}\left(\mathbb{R}^{d}\right)\right)}^{2}\|\bar{h}\|_{\mathcal{H}}^{2}=\|T(\bar{h})\|_{L^{p}\left(\mathbb{R}^{d}\right)}^{2} .
$$

On the other hand by (2.1) we can deduce

$$
\|T\|_{\mathcal{L}\left(\mathcal{H}, L^{p}\left(\mathbb{R}^{n}\right)\right)}^{2} \leq \frac{\left(\|T\|_{\mathcal{L}\left(\mathcal{H}, L^{p}\left(\mathbb{R}^{n}\right)\right)}^{2}\|\bar{h}\|_{\mathcal{H}}^{2}+\left\|T\left(h_{n}-\bar{h}\right)\right\|_{L^{p}\left(\mathbb{R}^{n}\right)}^{2}+o(1)\right)}{\left\|h_{n}-\bar{h}\right\|_{\mathcal{H}}^{2}+\|\bar{h}\|_{\mathcal{H}}^{2}+o(1)}
$$

and we easily get

$$
\begin{aligned}
& \|T\|_{\mathcal{L}\left(\mathcal{H}, L^{p}\left(\mathbb{R}^{d}\right)\right)}^{2}\left(\left\|h_{n}-\bar{h}\right\|_{\mathcal{H}}^{2}+o(1)\right) \\
& \leq\left(\left\|T\left(h_{n}-\bar{h}\right)\right\|_{L^{p}\left(\mathbb{R}^{d}\right)}^{2}+o(1)\right) .
\end{aligned}
$$

Notice that either $\left\|h_{n}-\bar{h}\right\|_{\mathcal{H}}=o(1)$ (and in this case we can conclude) or (up to subsequence)

$$
\inf _{n \in \mathbb{N}}\left\|h_{n}-\bar{h}\right\|_{\mathcal{H}} \geq \epsilon_{0}>0
$$

In particular by (2) we get

$$
\left.\|T\|_{\mathcal{L}\left(\mathcal{H}, L^{p}\left(\mathbb{R}^{d}\right)\right)}^{2} \leq\left\|T\left(\frac{h_{n}-\bar{h}}{\left\|h_{n}-\bar{h}\right\|_{\mathcal{H}}}\right)\right\|_{L^{p}\left(\mathbb{R}^{d}\right)}^{2}+o(1)\right)
$$

which by definition of $\|T\|_{\mathcal{L}\left(\mathcal{H}, L^{p}\left(\mathbb{R}^{d}\right)\right)}$ necessarily implies

$$
\left.\|T\|_{\mathcal{L}\left(\mathcal{H}, L^{p}\left(\mathbb{R}^{d}\right)\right)}^{2}=\left\|T\left(\frac{h_{n}-\bar{h}}{\left\|h_{n}-\bar{h}\right\|_{\mathcal{H}}}\right)\right\|_{L^{p}\left(\mathbb{R}^{d}\right)}^{2}+o(1)\right)
$$

and equivalently

$$
\left.\|T\|_{\mathcal{L}\left(\mathcal{H}, L^{p}\left(\mathbb{R}^{d}\right)\right)}^{2}\left\|h_{n}-\bar{h}\right\|_{\mathcal{H}}^{2}=\left\|T\left(h_{n}-\bar{h}\right)\right\|_{L^{p}\left(\mathbb{R}^{d}\right)}^{2}+o(1)\right) .
$$

By combining the first identity in (2.1) with (2.3) and (2.6) we get

$$
1=\frac{\left(\left\|h_{n}-\bar{h}\right\|_{\mathcal{H}}^{p}+\|\bar{h}\|_{\mathcal{H}}^{p}+o(1)\right)^{\frac{2}{p}}}{\left\|h_{n}-\bar{h}\right\|_{\mathcal{H}}^{2}+\|\bar{h}\|_{\mathcal{H}}^{2}+o(1)}
$$

i.e.

$$
\left(\left\|h_{n}-\bar{h}\right\|_{\mathcal{H}}^{p}+\|\bar{h}\|_{\mathcal{H}}^{p}\right)^{\frac{2}{p}}=\left\|h_{n}-\bar{h}\right\|_{\mathcal{H}}^{2}+\|\bar{h}\|_{\mathcal{H}}^{2}+o(1) .
$$

Since we are assuming $p \in(2, \infty)$ it is easy to deduce by a convexity argument that the previous inequality implies $\|\bar{h}\|_{\mathcal{H}}=1$ and $\left\|h_{n}-\bar{h}\right\|_{\mathcal{H}}^{2}=o(1)$ (actually we have excluded the possibility $\|\bar{h}\|_{\mathcal{H}}=0$ and $\left\|h_{n}-\bar{h}\right\|_{\mathcal{H}}^{2}=1+o(1)$ since by assumption $\bar{h} \neq 0)$.

\section{Proof of Thm 0.1}

\section{The case $p \neq \infty$}

Let $\hat{h}_{n} \in L^{2}(d \mu)$ be a maximizing sequence for $T_{\mu}$ w.r.t. $p$ (where $p$ is as in the assumptions).

First step: there is a sequence $x_{n} \in \mathbb{R}^{d}$ such that $\hat{g}_{n}(\xi)=e^{i x_{n} \cdot \xi} \hat{h}_{n}(\xi)$ has a weak limit different from zero in $L^{2}(d \mu)$ 
In order to verify this property we prove that there is $x_{n}$ such that

$$
T_{\mu}\left(\left(e^{i x_{n} \cdot \xi} \hat{h}_{n}(\xi)\right)=\tau_{x_{n}} T_{\mu}\left(\hat{h}_{n}(\xi)\right)\right.
$$

has a weak limit different from zero (here $\tau_{y}$ denotes the translation of vector $y$ ). Notice that by definition we have

$$
\left\|T_{\mu} \hat{h}_{n}\right\|_{L^{p}\left(\mathbb{R}_{x}^{n}\right)} \rightarrow\left\|T_{\mu}\right\|_{\mathcal{L}\left(L^{2}(d \mu), L^{p}\left(\mathbb{R}_{x}^{n}\right)\right)}>0 .
$$

By using the $(R C)_{\bar{p}}$ condition for a suitable $p_{0}(\mu)<\bar{p}<p$ we get

$$
\left\|T_{\mu} \hat{h}_{n}\right\|_{L^{\bar{p}}\left(\mathbb{R}_{x}^{n}\right)} \leq\|T\|_{\mathcal{L}\left(L^{2}(d \mu), L^{\bar{p}}\left(\mathbb{R}_{x}^{n}\right)\right)}\left\|\hat{h}_{n}(\xi)\right\|_{L^{2}(d \mu)}
$$

and hence

$$
\sup _{n \in \mathbb{N}}\left\|T_{\mu} \hat{h}_{n}\right\|_{L^{\bar{p}}\left(\mathbb{R}_{x}^{d}\right)} \equiv S<\infty .
$$

Next notice that we have the following inequality:

$$
\left\|T_{\mu} \hat{h}_{n}\right\|_{L^{p}\left(\mathbb{R}_{x}^{d}\right)} \leq\left\|T_{\mu} \hat{h}_{n}\right\|_{L^{\bar{p}}\left(\mathbb{R}_{x}^{d}\right)}^{\theta}\left\|T_{\mu} \hat{h}_{n}\right\|_{L^{\infty}\left(\mathbb{R}_{x}^{d}\right)}^{1-\theta}
$$

where $\frac{1}{p}=\frac{\theta}{\bar{p}}$. By combining this fact with (2.8) and (2.9) we deduce

$$
\left\|T_{\mu} \hat{h}_{n}\right\|_{L^{\infty}\left(\mathbb{R}_{x}^{d}\right)} \geq \epsilon_{0}>0 .
$$

Notice also that we have (by compactness of the support of $d \mu$ )

$$
\left\|T_{\mu} \hat{h}_{n}\right\|_{L^{\infty}\left(\mathbb{R}_{x}^{d}\right)} \leq\|\hat{h}\|_{L^{2}(d \mu)} \sqrt{\|d \mu\|}
$$

and

$$
\begin{gathered}
\left\|\nabla_{x} T_{\mu} \hat{h}_{n}\right\|_{L^{\infty}\left(\mathbb{R}_{x}^{d}\right)}=\left\|T_{\mu}\left(i \xi \hat{h}_{n}\right)\right\|_{L^{\infty}\left(\mathbb{R}_{x}^{d}\right)} \\
\leq\left\|\xi \hat{h}_{n}\right\|_{L^{2}} \sqrt{\|d \mu\|} \leq \sqrt{\|d \mu\|}\left(\sup _{\xi \in \operatorname{supp}(\mu)}|\xi|\right)\left\|\hat{h}_{n}\right\|_{L^{2}(d \mu)}
\end{gathered}
$$

(where $\|d \mu\|=\int 1 d \mu$ ). Hence

$$
\sup _{n \in \mathbb{N}}\left\|T_{\mu} \hat{h}_{n}\right\|_{W^{1, \infty}\left(\mathbb{R}_{x}^{d}\right)}<\infty .
$$

By (2.10) there exist $x_{n}$ such that

$$
\left|T_{\mu} \hat{h}_{n}\left(x_{n}\right)\right| \geq \epsilon_{0}>0
$$

and hence

$$
\left|\tau_{x_{n}} T_{\mu} \hat{h}_{n}(0)\right| \geq \epsilon_{0}>0 .
$$

On the other hand by (2.11) we get

$$
\left\|\tau_{x_{n}} T_{\mu} \hat{h}_{n}\right\|_{W^{1, \infty}(B(0,1))}
$$

are uniformly bounded and hence by the Ascoli-Arzelá Theorem

$$
\tau_{x_{n}}\left(T_{\mu} \hat{h}_{n}(\xi)\right)
$$

has an uniform limit in $B(0,1)$. By $(2.12)$ the limit has to be different from zero. Second step: conclusion of the proof

Notice that

$$
\left\|\hat{g}_{n}(\xi)\right\|_{L^{2}}=1
$$


and

$$
\left\|T_{\mu}\left(\hat{g}_{n}\right)\right\|_{L^{p}\left(\mathbb{R}_{x}^{n}\right)}=\left\|T_{\mu}\left(\hat{h}_{n}\right)\right\|_{L^{p}\left(\mathbb{R}_{x}^{n}\right)} .
$$

Hence $\hat{g_{n}}$ is a maximizing sequence for $T_{\mu}$. On the other hand by the previous step it is easy to check that all the hypothesis of Proposition 0.1 are satisfied if we choose $T=T_{\mu}, \mathcal{H}=L^{2}(d \mu)$ and we fix as a maximizing sequence $\hat{g}_{n}$.

The case $p=\infty$

Following the computations done above we have that

$$
\lim _{n \rightarrow \infty}\left\|T_{\mu} \hat{h}_{n}\right\|_{L^{\infty}\left(\mathbb{R}_{x}^{n}\right)}=\left\|T_{\mu}\right\|_{\mathcal{L}\left(L^{2}(d \mu), L^{\infty}\left(\mathbb{R}_{x}^{d}\right)\right)}
$$

and moreover

$$
\sup _{n \in \mathbb{N}}\left\|T_{\mu} \hat{h}_{n}\right\|_{W^{1, \infty}\left(\mathbb{R}_{x}^{d}\right)}<\infty .
$$

In particular there is a sequence $x_{n} \in \mathbb{R}^{d}$ such that

$$
\lim _{n \rightarrow \infty}\left\|T_{\mu} \hat{h}_{n}\left(x_{n}\right)\right\|=\left\|T_{\mu}\right\|_{\mathcal{L}\left(L^{2}(d \mu), L^{\infty}\left(\mathbb{R}_{x}^{d}\right)\right)} .
$$

As in the previous case we introduce $\hat{g}_{n}=e^{i x_{n} \cdot \xi} \hat{h}(\xi)$ and it is easy to deduce that $\hat{g}_{n}$ is still maximizing sequence with the extra property that

$$
\lim _{n \rightarrow \infty}\left|T_{\mu}\left(\hat{g}_{n}\right)(0)\right|=\left\|T_{\mu}\right\|_{\mathcal{L}\left(L^{2}(d \mu), L^{\infty}\left(\mathbb{R}_{x}^{d}\right)\right)} .
$$

By the Ascoli-Arzelá theorem (that can be applied due to (2.13)) in conjunction with (2.14) we conclude that if $\bar{g}$ is the the weak limit of $\hat{g}_{n}$ in $L^{2}(d \mu)$ then necessarily

$$
\left\|T_{\mu}(\bar{g})\right\|_{L^{\infty}\left(\mathbb{R}_{x}^{n}\right)} \geq\left|T_{\mu} \bar{g}(0)\right|=\left\|T_{\mu}\right\|_{\mathcal{L}\left(L^{2}(d \mu), L^{\infty}\left(\mathbb{R}_{x}^{d}\right)\right)} .
$$

On the other hand by semicontinuity of the norm $L^{2}(d \mu)$ we have that $\|\bar{g}\|_{L^{2}(d \mu)} \leq$ 1. By combining this fact with the definition of $\left\|T_{\mu}\right\|_{\mathcal{L}\left(L^{2}(d \mu), L^{\infty}\left(\mathbb{R}_{x}^{d}\right)\right)}$ we easily deduce that $\|\bar{g}\|_{L^{2}(d \mu)}=1$ and hence $\hat{g}_{n}$ is compact in $L^{2}(d \mu)$.

\section{REFERENCES}

[1] H.Brézis, E. Lieb A relation between pointwise convergence of functions and convergence of functionals. Proc. Amer. Math. Soc. 88 (1983), no. 3, 486-490.

[2] M. Christ, S. Shao Existence of Extremals for a Fourier Restriction Inequality. arXiv: $1006.4319 \mathrm{v} 1$

[3] D. Foschi Maximizers for the Strichartz inequality. J. Eur. Math. Soc. (JEMS) 9 (2007), no. $4,739-774$.

[4] E. Lieb Sharp constants in the Hardy-Littlewood-Sobolev and related inequalities. Ann. of Math. (2) 118 (1983), no. 2, 349-374.

Luca Fanelli: Universidad del Pais Vasco, Departamento de Matemáticas, Apartado 644, 48080, Bilbao, Spain

E-mail address: luca.fanelli@ehu.es

Luis Vega: Universidad del Pais Vasco, Departamento de Matemáticas, Apartado 644, 48080, Bilbao, Spain

E-mail address: luis.vega@ehu.es

Nicola Visciglia: Universitá di Pisa, Dipartimento di Matematica, Largo B. PonteCoRvo 5, 56100 Pisa, Italy

E-mail address: viscigli@dm.unipi.it 